\title{
MS.liverK: an R package for transcriptome-based computation of molecular subtypes and functional signatures in liver cancer
}

Florent Petitprez ${ }^{1,2,3,4}$, Léa Meunier ${ }^{5}$, Eric Letouzé ${ }^{5}$, Yujin Hoshida ${ }^{6}$, Augusto Villanueva ${ }^{6,7}$, Josep Llovet ${ }^{6,8}$, Snorri Thorgeirsson ${ }^{9}$, Xin Wei Wang ${ }^{9}$, Wolf $\mathrm{H}$ Fridman $^{2,3,4}$, Jessica Zucman-Rossi ${ }^{5,10}$ and Aurélien de Reyniès ${ }^{1, *}$

${ }^{1}$ Programme Cartes d'Identité des Tumeurs (CIT), Ligue Nationale Contre le Cancer, Paris, France

${ }^{2}$ INSERM UMR_S 1138, Cordeliers Research Centre, Paris, France

${ }^{3}$ Paris Descartes University, Sorbonne Paris Cité, Paris, France

${ }^{4}$ Pierre and Marie Curie University, Paris, France

${ }^{5}$ INSERM UMR-1162, Paris, France; 'Liver Cancer Program, Division of Liver Diseases, Department of Medicine, Tisch Cancer Institute, Icahn School of Medicine at Mount Sinai, New York, NY, USA

${ }^{7}$ Division of Haematology and Medical Oncology, Department of Medicine, Tisch Cancer Institute, Icahn School of Medicine at Mount Sinai, New York, NY, USA

${ }^{8}$ BCLC, Liver Unit, IDIBAPS, CIBERehd, Hospital Clínic, Universitat de Barcelona, ICREA, Barcelona, Catalonia, Spain

9Laboratory of Human Carcinogenesis, CCR, NCI, NIH, Bethesda, MD, USA

${ }^{10} \mathrm{AP}-\mathrm{HP} ;$ HEGP, Department of Oncology, Paris, France.

*To whom correspondence should be addressed: reyniesa@ligue-cancer.net.

\section{Abstract}

\section{Abstract}

Summary: Liver cancer is a highly heterogeneous disease in terms of etiology, tissue and cellular morphology, tumor molecular characteristics, microenvironment composition and prognosis. Several studies, based on tumor gene-expression profiling (GEP) data, have dissected the molecular heterogeneity of liver cancer. They resulted in various tools, either delineating homogeneous tumor subtypes or calculating molecular scores of prognostic or biological functions. Here, we present MS.liverk, an easy-to-use R package providing a comprehensive implementation of these tools, for research use.

Availability and implementation: The MS.liverk $\mathrm{R}$ package is available from GitHub (https://github.com/cit-bioinfo/MS.liverK).

\section{Introduction}

Primary liver cancer is the third most deadly cancer worldwide, with Hepatocellular carcinomas (HCC) accounting for $85 \%-90 \%$ of the cases. The molecular heterogeneity of liver cancers has motivated numerous transcriptome-based studies, yielding gene-signatures and algorithms for molecular subtyping, prognostic prediction and functional scores computation. The use of these tools is of great interest for researchers dedicated to liver cancer study, but is very limited due to their dispersion.

Here, we introduce MS.liverk (Molecular Signatures in Liver Cancer), an R package reimplementing these tools. MS.liverK takes as input a (log2 scale) transcriptome matrix of liver cancer samples, either microarray- or RNA-Seq-derived. It also includes graphical functions, allowing to easily visualize the outputs. 


\section{MS.liverK}

After a careful review of the literature, we identified 5 GEP-based molecular subtyping systems of HCCs published in the last 15 years (Lee et al., 2004; Boyault et al., 2007; Yamashita et al., 2008; Chiang et al., 2008; Hoshida et al., 2009). Lee classification (Lee et al., 2004) was established on a belgo-chinese cohort of $91 \mathrm{HCC}$ samples; it is made of 2 classes (A/B), related to proliferation and survival. Lee subtypes have been further refined in 4 groups using Alpha-fetoprotein (AFP) marker, improving association to survival. Boyault classification (Boyault et al., 2007) was established on a French cohort of $57 \mathrm{HCCs}$ including patients from various geographic origins; it is made of 6 classes (G1 to G6) related to TP53 and CTNNB1 mutations, proliferation, HBV infection and prognosis. Yamashita classification (Yamashita et al., 2008) is based on two markers, EPCAM and AFP, and defines four groups related to differentiation and prognosis. Chiang classification (Chiang et al., 2008) was built using 91 HCV infected HCCs; it identifies 5 classes related to CTNNB1 mutation, proliferation, inflammation and polysomy of the chromosome 7. Hoshida classification (Hoshida et al., 2009) is based on the meta-analysis of 9 cohorts totaling $603 \mathrm{HCC}$ samples; it contains 3 classes (S1/S2/S3) related to proliferation, differentiation and survival. MS.liverk implements all of the above classification systems from transcriptomic data; it also includes a conversion function to allow using different GEP platforms (Fig. S1A).

To ensure classifiers were adequately assigning classes to samples, we ran them on the data from the samples used by the four teams to define their subgroups. We then compared MS.liverk classes to the ones they originally established and found a very good correspondence (Fig. S1B): Chi-squared test pvalues for Lee, Boyault, Chiang and Hoshida and Roessler classifications were all $<2.2$ e-16, with accuracy rate of respectively $94.5 \%, 98.2 \%, 100 \%, 93.8 \%$ and $90.0 \%$.

MS.liverk implements the prognostic prediction algorithm published by (Nault et al., 2013), which is so far the most extensively validated prognostic score for hepatocellular carcinomas (HCC).

Several teams have published gene-signatures related to biological functions/pathways involved in liver cancer oncogenesis. These gene-signatures relate to TGFB1 signalling (Coulouarn et al., 2008), MET pathway (Kaposi-Novak et al., 2006), stemness (Oishi et al., 2012; Yamashita et al., 2008), EPCAM (Yamashita et al., 2008) and hypoxia (van Malenstein et al., 2010). MS.liverK computes all the corresponding scores.

We applied MS.liverK to the TCGA LIHC RNA-seq dataset $(n=286)$. The graphical output shows a striking concordance across the various subtyping systems, the prognostic score and the functional scores (Figure 1). This observation is expected, given that molecular subtypes should represent homogeneous types of tumors with specific biological and prognostic characteristics.

\section{Software implementation and use}

The package has been entirely written using $R$ language. It includes pre-packaged data from Gene Expression Omnibus (GEO), accession number GSE20238 (Mínguez et al., 2011), to be used as example data. A vignette describing the use of the package is available on GitHub (https://github.com/FPetitprez/MS.liverK/blob/master/vignettes/vignette.pdf). On a standard computer, the computation of all subtypes and scores takes a couple of minutes. 


\section{References}

Boyault,S. et al. (2007) Transcriptome classification of HCC is related to gene alterations and to new therapeutic targets. Hepatology, 45, 42-52.

Chiang,D.Y. et al. (2008) Focal gains of VEGFA and molecular classification of hepatocellular carcinoma. Cancer Res. , 68, 6779-6788.

Coulouarn,C. et al. (2008) Transforming growth factor-beta gene expression signature in mouse hepatocytes predicts clinical outcome in human cancer. Hepatology, 47, 2059-2067.

Hoshida,Y. et al. (2009) Integrative transcriptome analysis reveals common molecular subclasses of human hepatocellular carcinoma. Cancer Res., 69, 7385-7392.

Kaposi-Novak,P. et al. (2006) Met-regulated expression signature defines a subset of human hepatocellular carcinomas with poor prognosis and aggressive phenotype. J. Clin. Invest., 116, 15821595.

Lee,J.-S. et al. (2004) Classification and prediction of survival in hepatocellular carcinoma by gene expression profiling. Hepatology, 40, 667-676.

van Malenstein,H. et al. (2010) A seven-gene set associated with chronic hypoxia of prognostic importance in hepatocellular carcinoma. Clin. Cancer Res., 16, 4278-4288.

Mínguez,B. et al. (2011) Gene-expression signature of vascular invasion in hepatocellular carcinoma. J. Hepatol., 55, 1325-1331.

Nault,J.-C. et al. (2013) A hepatocellular carcinoma 5-gene score associated with survival of patients after liver resection. Gastroenterology, 145, 176-187.

Oishi,N. et al. (2012) Transcriptomic profiling reveals hepatic stem-like gene signatures and interplay of miR-200c and epithelial-mesenchymal transition in intrahepatic cholangiocarcinoma. Hepatology, 56, 1792-1803.

Yamashita,T. et al. (2008) EpCAM and alpha-fetoprotein expression defines novel prognostic subtypes of hepatocellular carcinoma. Cancer Res., 68, 1451-1461. 


\section{Molecular subtypes}

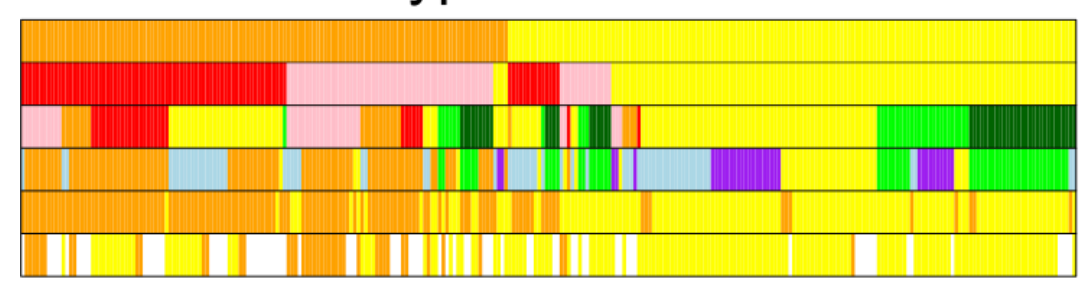

\section{Prognosis}

\section{Functional scores}

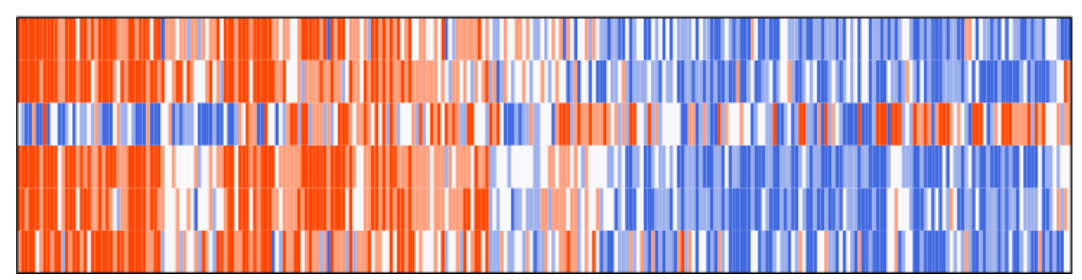

$\begin{array}{lcc}\text { Lee } & \text { Lee, Roessler } & \text { Hoshida } \\ \text { Hoshida } & \square \text { A } & \square \text { S1 } \\ \text { Boyault } & \square \mathrm{B} & \square \mathrm{S} 2 \\ \text { Chiang } & \text { Boyault } & \square \mathrm{S3} \\ \text { Roessler } & \square \mathrm{G} 1 & \text { Chiang } \\ \text { EPCAM.AFP } & \square \mathrm{G} 2 & \square \text { CTNNB1 } \\ & \square \mathrm{G} 3 & \square \text { Inflammation } \\ & \square \text { G } & \square \text { Polysomy chr7 } \\ & \square \text { G5 } & \square \text { Proliferation } \\ & \square \text { G6 } & \square \text { Unannotated }\end{array}$

Nault

Fig. 1. MS.liverk graphical output. Graphical output of MS.liverK illustrated on the TCGA LIHC transcriptome dataset. Columns represent samples, rows represent molecular subtypes (upper panel), prognostic scores (middle panel) and functional scores (lower panel).

Coulouarn_temporal_TGFB1_signature Kaposi_liverK_met Oishi_Cholangio_stem_cell_like Yamashita_liverK_stem_cell Yamashita_liverK_with_EPCAM Van_Malenstein_hypoxia 